\title{
Planar biaxial testing of soft biological tissue using rakes: a critical analysis of protocol and fitting process
}

\author{
Heleen Fehervary*, Marija Smoljkić, Jos Vander Sloten, Nele Famaey \\ Biomechanics Section, KU Leuven, Leuven, Belgium
}

\begin{abstract}
Mechanical characterization of soft biological tissue is becoming more and more prevalent. Despite the growing use of planar biaxial testing for soft tissue characterization, testing conditions and subsequent data analysis have not been standardized and vary widely. This also influences the quality of the result of the parameter fitting. Moreover, the testing conditions and data analysis are often not or incompletely reported, which impedes the proper comparison of parameters obtained from different studies.

With a focus on planar biaxial tests using rakes, this paper investigates varying testing conditions and varying data analysis methods and their effect on the quality of the parameter fitting results. By means of a series of finite element simulations, aspects such as number of rakes, rakes' width, loading protocol, constitutive model, material stiffness and anisotropy are evaluated based on the degree of homogeneity of the stress field, and on the correlation between the experimentally obtained stress and the stress derived from the constitutive model. When calculating the aforementioned stresses, different definitions of the section width and deformation gradient are used in literature, each of which are looked into. Apart from this degree of homogeneity and correlation, also the effect on the quality of the parameter fitting result is evaluated.

The results show that inhomogeneities can be reduced to a minimum for
\end{abstract}

\footnotetext{
* Corresponding author: H. Fehervary, KU Leuven, Biomechanics Section, Celestijnenlaan 300C, 3001 Heverlee (Belgium)

Email address: heleen.fehervary@kuleuven.be (Heleen Fehervary )
}

Preprint submitted to Journal of the Mechanical Behavior of Biomedical MaterialsJanuary 14, 2016 
wise choices of testing conditions and analysis methods, but never completely eliminated. Therefore, a new parameter optimization procedure is proposed that corrects for the inhomogeneities in the stress field and induces significant improvements to the fitting results. Recommendations are made for best practice in rake-based planar biaxial testing of soft biological tissues and subsequent parameter fitting, and guidelines are formulated for reporting thereof in publications.

Keywords: constitutive modelling, planar biaxial testing, parameter fitting, inhomogeneity, FE simulations

\section{Introduction}

The performance of biomedical devices and procedures is increasingly assessed by means of finite element (FE) simulations [1, 2]. Logically, using appropriate material properties is crucial for the outcome of the simulation. Hence, apart from elucidating relative trends between tissue types, the absolute characterization of the mechanical behaviour of biological tissue is gaining importance.

Mechanical characterization of soft biological tissue through planar biaxial testing is becoming more and more prevalent [3, 4, 5, 6] and is used for all kinds of nearly-incompressible thin tissues.

Three types of gripping mechanisms are commonly used (see Figure 1): clamps, rakes, and sutures (also referred to as tethers). None of these result in a homogeneous stress state [7, the biggest confounding factors being discontinuous loading and unknown contra-lateral forces. Rigidly clamped specimens are loaded most continuously, but this method induces substantial unmeasured contra-lateral forces which are a.o. geometry- and displacement-dependent 8 . Nolan et al. recently suggested that an inverse FE-based method is the only way to account for these contra-lateral forces in the case of non-linear anisotropic materials 8 . These contra-lateral forces create a stress shield for the region of interest [7] causing an artificial increase in the apparent stiffness [9, 10. This 
stress shielding effect is typically reduced with cruciform-shaped samples. However, these imply substantial tissue loss during sample preparation, a luxury one can generally not afford when testing biological tissues [3].

On the other end of the spectrum, sutures do allow contra-lateral movement and rotation of the sample, and are typically used with square samples. Suturebased systems with pulleys, as shown e.g. in [11] are designed such that no shear stress is transferred onto the sample, and are therefore especially fit to deliberately induce shear deformation in samples with material axes that are rotated w.r.t. the testing axes. Deliberately inducing shear can be required when the material axes are an unknown parameter that require fitting, or when the material model contains specific terms activated only in shear. However, attaching sutures to the specimen is highly unrepeatable, even if a template is used, and results in a non-uniform application of the load.

Rake-based systems do transfer a limited amount of contra-lateral forces onto the sample and are less fit for large shear deformations, but are substantially easier to mount in a repeatable and equally spaced manner. Hence, although the applied load is still discontinuous, using rakes results in a more uniform load distribution. Therefore, when testing samples with symmetric material axes that can be aligned with the testing axes, and fitting them to material models that do not contain specific shear terms, rake-based systems are often the method of choice.

Each of the above testing methods requires a different approach and results in different stress states. This paper does not provide an in depth discussion of the benefits and drawbacks of each method, but focuses rather on the specific case of rake-based planar biaxial testing of a square sample.

Despite the growing use of planar biaxial testing, protocols have not been standardized and methodologies vary widely [7]. One explanation is the difficult validation of testing protocols and fitting processes, since the ground truth, i.e. the 'correct' material parameters of a certain tissue, is unknown. This is due to the fact that also the constitutive material models for which parameters are sought, are approximations of reality. 
Constitutive models represent the mechanical behaviour of biological tissues with a mathematical expression that relates stress to strain. The material parameters can be determined through a parameter fitting procedure by comparing the model stresses to the stresses obtained in a mechanical experiment, referred to as experimental stresses. In the optimization process, the material parameters of the constitutive model are altered iteratively until the model stresses match the experimental stresses as good as possible [12.

The quality of the result of the parameter fitting is influenced by different aspects affecting the experimental and model stresses. First of all, the experimental stress value is generally obtained assuming a homogeneous stress field [13. As mentioned earlier, depending on the gripping mechanism and the specimen shape used (Figure 1), this assumption is true to a greater or lesser extent. While the effect of these boundary conditions on the homogeneity of the stressstrain field has been investigated before [10, 7, 9, 14, the effect on the parameter fitting is less clear.

Apart from the gripping mechanism, also the selected constitutive model, the definition of the deformation gradient, the section surface and other geometrical parameters can strongly influence the parameter fitting result. Nevertheless, current practice of planar biaxial tensile tests for soft biological tissues seems to be lacking consensus on these aspects and, often, choices made in the experiment and analysis process influencing the outcome are not or incompletely reported. This impedes the proper comparison of parameters obtained from different studies. Therefore, this paper will elaborate on the effect of these modelling aspects and choices regarding the experimental set-up on the stress-strain field and on the result of the parameter fitting. This knowledge can then lead to recommendations for best practice in planar biaxial testing using rakes and subsequent parameter fitting, as well as to guidelines for reporting thereof in publications.

To quantitatively assess the aspects discussed above, a FE simulation of a planar biaxial test using rakes as a gripping mechanism is employed. This corresponds to an ideal biaxial experimental situation, in which noise introduced by practical inaccuracies and sensors is absent and the ground truth, i.e. the 
material parameters assigned to the model, is known. In the following sections, first the basic FE model is described, followed by a section explaining the calculation of the model and experimental stresses. The next sections provide an overview of variations to the experimental set-up, to the stress calculations and to the parameter fitting procedure. Subsequently the results of these FE simulations and the data analysis are presented and discussed. Finally, conclusions are formulated as recommendations for best practice and reporting of planar biaxial testing using rakes.

\section{Materials and Methods}

\subsection{FE simulation: Basic model}

A FE model is built in Abaqus/Standard 6.12-2 and simulates a displacementcontrolled planar biaxial test of a square specimen of human abdominal aorta using rakes. Due to material and set-up symmetry, only a quarter of the testing specimen needs to be simulated (see Figure 2). The dimensions of this quarter are $3.5 \mathrm{~mm}$ by $3.5 \mathrm{~mm}$. Displacements are imposed on the rakes' attachment sites at two adjacent sides of the specimen. Symmetry boundary conditions are imposed on the other sides of the specimen (see Figure 3). The boundary and loading conditions allow contra-lateral movement of the specimen, as in an actual biaxial experiment using rakes. The rakes themselves are not modelled. The rakes are loaded mainly along their length axis, however a small lateral bending force is also induced when the rakes move laterally. As the rakes are not modelled in this FE model, this implies that the lateral movement of the sample at the rakes' attachment sites is slightly overestimated. However, at the center of the sample and at the marker location, this overestimation is negligible (see section 2.5.1 for a second FE simulation that does incorporate rakes). The normal forces at the rakes' attachment sites resulting from the applied displacement and the displacements of a virtual marker are tracked. The marker is placed such that it is located in the central region of the entire specimen. It consists of an average of four nodes in cruciform spaced $0.3 \mathrm{~mm}$ apart. 
The specimens used in biaxial experiments are thin, allowing the assumption of no shear through the thickness (i.e. a plane stress state) and the use of shell elements, specifically S4R. Shell elements have a similar topology as plane stress elements, but offer a computational benefit. Reduced integration also offers a computational benefit and is used by default in Abaqus for shell elements in combination with a hyperelastic material. It is justified since we are interested in the overall behaviour of the sample. The mesh consists of S4R elements and is shown in Figure 3. A mesh convergence analysis determined that for 16502 elements the convergence error of the correction factor was smaller than $1 \%$. The convergence error of the correction factor (described further in section 2.3.1 is defined as the difference between a model with $n$ elements and a model with $2 n$ elements in the ratio $\sigma_{\bmod } / \sigma_{\text {exp }}$ [15. Incompressibility is enforced by setting the bulk modulus $D_{1}=0$.

Data is exported as in an actual biaxial experiment: normal forces at the rakes and plane displacements of the marker are tracked. The same number of time data points is used in every simulation. With this data and with a known material model and parameters, both the experimental and the model stresses are calculated as explained in section 2.2 .

As detailed below, the FE simulation described is repeated for variations to the experimental set-up. Also variations to the stress calculations and to the parameter fitting procedure are made. As such, the influence of different parameters, definitions, assumptions and fitting procedures is investigated.

\subsection{Model and experimental stress calculation}

In planar biaxial tensile tests using rakes, forces and hence stresses can be acquired in two perpendicular directions, while displacements are symmetrically applied along the same directions. Hence, the objective function to be minimized is:

$$
\left(\boldsymbol{\sigma}_{11}^{\text {mod }}-\boldsymbol{\sigma}_{11}^{e x p}\right)^{2}+\left(\boldsymbol{\sigma}_{22}^{\text {mod }}-\boldsymbol{\sigma}_{22}^{e x p}\right)^{2}
$$


Experimental stresses $\boldsymbol{\sigma}_{i i}^{e x p}$ are calculated using the force applied at the gripping mechanism and a section surface, whereas model stresses $\boldsymbol{\sigma}_{i i}^{\bmod }$ are calculated based on the constitutive model and the deformation tensor, where the latter is also derived from the experiments.

\subsubsection{Model stress calculation}

Model stresses are calculated based on the deformation gradient tensor and on the constitutive model. When the constitutive model is described with a strain energy density function (SEDF), the second Piola-Kirchhoff model stresses $\boldsymbol{S}_{m o d}$ are calculated as the derivatives of the $\operatorname{SEDF} \Psi$ with respect to the right Cauchy-Green tensor $C$ [16, 17]:

$$
\boldsymbol{S}_{m o d}=-p \boldsymbol{C}^{-1}+2 \frac{\partial \Psi(\boldsymbol{C})}{\partial \boldsymbol{C}}
$$

where $p$ is introduced for incompressible materials and serves as an indeterminate Lagrangian multiplier which can be interpreted as a hydrostatic pressure. $p$ can be calculated from the boundary condition: $\sigma_{m o d, 33}=0$. The Cauchy model stresses are then calculated by multiplying the second Piola-Kirchhoff stresses with the deformation gradient tensor $\boldsymbol{F}$ as follows: [16, 17]:

$$
\boldsymbol{\sigma}_{m o d}=\boldsymbol{F} \boldsymbol{S}_{m o d} \boldsymbol{F}^{T}
$$

The calculation of $\boldsymbol{F}$ is further explained in section 2.5.1.

\subsubsection{Experimental stress calculation}

Experimental stresses are calculated based on the force applied at the gripping mechanism and a section surface. The nominal stress or first Piola-Kirchhoff stress $\boldsymbol{P}$ is calculated as:

$$
P_{\text {exp }, i i}=\frac{F_{i}}{A_{i, 0}} \quad i=x, y .
$$

With $F_{i}$ the load applied at the rakes in the $i$-th direction and $A_{i, 0}$ the undeformed section surface with normal in $i$-th direction. As the force is typically only measured in the direction of the test axes, $\boldsymbol{P}_{\exp }$ is a diagonal matrix. This 
direction will remain constant throughout the experiment as rakes do not allow small body rotations. In contrast, a suture-based planar biaxial set-up uses a pulley system that allows small body rotations. These small body rotations should be taken into account, for example with the method proposed in [11.

The relation between the first Piola-Kirchhoff stress and Cauchy stress for incompressible materials is defined by the deformation gradient $\boldsymbol{F}$ [16, 17]:

$$
\boldsymbol{\sigma}_{\text {exp }}=\boldsymbol{P}_{\text {exp }} \boldsymbol{F}^{T}
$$

The undeformed section surface $A_{i, 0}$ is defined by the initial specimen thick-

ness $h_{0}$ and the initial section width $l_{i 0}$ in the i-th direction. The definition of $l_{i 0}$ is further explained in section 2.5 .2 .

$$
A_{i, 0}=h_{0} l_{i 0}
$$

\subsection{Quality measures}

The FE simulation described in section 2.1 is repeated for variations to the experimental set-up. Also variations to the model and experimental stress calculation method and to the parameter fitting procedure are made. In this section two quality measures are defined to compare these varying conditions. Optimal biaxial testing conditions should have (1) a good correspondence between model and experimental stresses and (2) a homogeneous stress field. The correction factor $f$ and the inhomogeneity measure $h$, defined below, reflect these criteria.

\subsubsection{Correction factor $f$}

In a planar biaxial experiment, the material parameters are determined by matching model and experimental stresses in a parameter fitting process. In the FE simulations described here, the actual material parameters are known and the experimental and the model stresses (calculated using Equations 5 and 3 ) can be compared, allowing to determine a correction factor which reflects the error resulting from an inhomogeneous stress and strain field and an incorrect section surface. 
The correction factor $f$ is determined in an optimization process with objective function:

$$
\left(\boldsymbol{\sigma}_{11}^{\text {model }}-f \boldsymbol{\sigma}_{11}^{\text {exp }}\right)^{2}+\left(\boldsymbol{\sigma}_{22}^{\text {model }}-f \boldsymbol{\sigma}_{22}^{\text {exp }}\right)^{2}
$$

In other words, the experimental stress is fitted on the model stress by means of a correction factor as shown in Figure 4

This optimization problem is solved in Matlab R2013a using the non-linear least squares (lsqnonlin) routine and the trust-region-reflective optimization algorithm. The closer $f$ is to 1 , the better the experimental and model stresses agree and the better the results of the parameter fitting will be.

\subsubsection{Inhomogeneity $h$}

The inhomogeneity $h$ of the stress field is quantified by means of a root mean square error (RMSE) between the average stress and the actual stress at each node:

$h=\frac{1}{2} \sum_{x, y} R M S E\left(\sigma_{k}, \sigma_{m e a n}\right)$ with $k \in$ all nodes covered by area between markers

$$
\sigma_{\text {mean }}=\frac{1}{k} \sum_{i=1}^{k} \sigma_{i}
$$

In planar biaxial testing, markers are often attached to the specimen to measure the deformation optically by means of a camera. In this case the region between the markers is considered to be homogeneous, therefore, the RMSE is calculated for all the nodes inside this region. Stresses at nodes are used instead of stresses at integration points, as they are volume-averaged values of the stresses at the integration points. The smaller $h$, the more homogeneous the central region between the markers is.

\subsection{Variations to the experimental set-up}

Three aspects of the testing conditions are looked into: sample geometry, loading protocol and material behaviour. Each of the variations will be compared by means of the correction factor $f$ and the inhomogeneity measure $h$. An overview of the variations is given in Table 4. 


\subsubsection{Geometry}

Different parameters define the geometry of a planar biaxial experiment. Those that are varied in the simulations are represented in Figure 5 number of rakes $\left(n_{r}\right)$, marker position $\left(L_{m}\right)$, rakes' width $\left(R_{w}\right)$ and apron size, defined as the difference between the sample length $\left(L_{s}\right)$ and length covered by the rakes $\left(L_{r}\right)$.

Number of rakes $n_{r}$. The number of rakes $n_{r}$ is varied from 3 to 6 . The ratio rakes' width $R_{w}$ - length covered by the rakes $L_{r}$ is thereby held constant, meaning that the inter-rake distance $R_{d}$ is adjusted for a different number of rakes. This allows a fair comparison between varying numbers of rakes.

$$
\frac{R_{w}}{L_{r}}=\frac{R_{d}\left(n_{r}-1\right)}{L_{r}}=0.8
$$

Marker position $L_{m}$. The position of the marker $L_{m}$ is varied from the centre of the specimen towards the rakes' attachment sites. Practically, the marker can not be placed exactly at the center because a certain distance to the other markers is necessary to be able to calculate the stretch. This limit is taken into account.

Rakes' width $R_{w}$. The rakes' width $R_{w}$ is altered by adjusting the inter-rakes distance $R_{d}$. The rakes' width is varied from covering a small to a large section of the rake surface, described by $L_{r}$.

Apron size $L_{s}-L_{r}$. The apron, i.e. the region outside of the rakes' attachment points, must be large enough to bear the applied loads without disproportionate deformation or material failure. The apron size is varied by altering the rake surface, described by the length covered by the rakes $L_{r}$, while the specimen surface, described by $L_{s}$, is held constant.

\subsubsection{Loading protocol}

Biaxial experiments are mostly force/tension- or displacement/strain-driven. Force/tension-driven protocol is more complex, since this is a closed-loop control 
process with coupling between both directions. A too simple implementation can lead to an unstable control. Displacement-control based on the displacement of the rakes is open-loop and therefore more straightforward. Note however, that in experimental practice, the actual displacement measured at the marker is lower than the imposed one as tear or slippage at the gripping mechanism occurs and gaps originate between the gripping mechanism and the tissue. In this series of FE simulations, a displacement-driven protocol is used. However, a force-controlled protocol would yield similar results, since tear or slippage phenomena were not taken into account in this idealized simulation.

A protocol of a biaxial experiment generally consists of multiple loading steps of increasing load and in each loading step different ratios between both testing directions. Both of these aspects are varied.

Ratios. Four different ratios are simulated: in the first three, the ratios between the circumferential and the axial stretch $\left(\lambda_{11}: \lambda_{22}\right)$ are as follows: $1: 1,0.5: 1$ and 1:0.5. The fourth one, 'Custom', has no fixed ratio and is meant to approximate the physiological loading condition. Therefore, the stretch in the axial direction $\lambda_{22}$ is constant, while the stretch in the circumferential direction $\lambda_{11}$ alters due to varying diastolic \& systolic pressures (e.g. as a consequence of hypertension).

Strain levels. The order of magnitude for the applied displacements is based on an estimate of the physiological circumferential and/or axial stretch with values from 18 and 19 . The strain levels $1,2,3$ and 4 correspond to $1 \times, 2 \times, 4 \times$ and $6 \times$ this estimate. The exact applied displacements differ according to the position of the rakes $L_{r}$. Therefore, the stretches are reported in Table 1 for each of the four ratios in every strain level.

\subsubsection{Material}

The material aspects that are varied are the following: constitutive model, material stiffness and fibre angle.

Constitutive model. The goal of the paper is not to discuss the performance of different constitutive models, but to generate an insight in the influence of a 
material on the results of a biaxial test. Therefore, two commonly used constitutive models are used in the simulations: the Gasser-Ogden-Holzapfel (GOH) material model [20] and the Neo-Hookean ( $\mathrm{NH}$ ) material model.

The GOH-material model is a structural constitutive model that describes an incompressible, fibre-reinforced and anisotropic material, that also accounts for dispersion of the fibres. The SEDF contains an isotropic and an anisotropic term, corresponding to the matrix material and to the collagen fibre families, respectively. With two fibre families, the SEDF is expressed as [20]:

$$
\Psi=C_{10}\left(I_{1}-3\right)+\sum_{i=4,6} \frac{k_{1}}{2 k_{2}}\left[e^{k_{2}\left(\kappa I_{1}+(1-3 \kappa) I_{i}-1\right)^{2}}-1\right]
$$

where $C_{10}>0$ and $k_{1}>0$ are stress-like parameters, respectively related to the matrix- and fibre-stiffness. $k_{2}>0$ is a dimensionless parameter and $\kappa \in\left[0, \frac{1}{3}\right]$ is a parameter related to the dispersion of the fibres, with the lower and upper limit corresponding to no fibre dispersion and fully dispersed fibres (i.e. isotropy), respectively.

$I_{1}$ is the first invariant of the right Cauchy-Green tensor $C$ and $I_{4}$ and $I_{6}$ are invariants of $\boldsymbol{C}$ related to the stretch in the mean direction of each fibre family. $C$ is a function of the deformation gradient.

$$
\begin{aligned}
& I_{1}=\operatorname{tr}(\boldsymbol{C}) \\
& I_{4}=\boldsymbol{m} \cdot(\boldsymbol{C m}) \\
& I_{6}=\boldsymbol{n} \cdot(\boldsymbol{C n}) \\
& \boldsymbol{C}=\boldsymbol{F}^{T} \boldsymbol{F}
\end{aligned}
$$

The fibre vectors $\boldsymbol{m}$ and $\boldsymbol{n}$ represents the mean fibre direction as a function of $\alpha$, here defined as the angle between the mean fibre direction and the longitudinal direction.

Besides the GOH-material model, the NH-material model is used. This 
constitutive model is isotropic and expresses the SEDF as:

$$
\Psi=C_{10}\left(I_{1}-3\right)
$$

$C_{10}$ is a material constant corresponding to the double of the shear modulus. $I_{1}$ is the first invariant of $\boldsymbol{C}$ and is calculated as in Equation 11a Note that the first term of the GOH-model is the NH-material model.

Stiffness. The mechanical behaviour is varied in the FE simulations by varying the material parameters. Table 2 and Table 3 show the different parameter sets for the GOH- and the NH-material model respectively. The NH-parameter sets 1, 2 and 3 were chosen such that the stresses of the NH-model were in the same range as the stresses of GOH-model (parameter set 1) for strain levels 1, 2 and 3 , respectively.

Fibre angle. The fibre angle $\alpha$, defined as the angle between the mean fibre direction and the longitudinal direction, is altered. Due to symmetry only values between 0 and $45^{\circ}$ are necessary: $0^{\circ}, 11.25^{\circ}, 22.5^{\circ}, 33.75^{\circ}$ and $45^{\circ}$. The other material parameters are as in GOH-parameter set 1 (see Table 2 ).

\subsection{Variations to the stress calculations}

As explained before, in a parameter fitting procedure, model and experimental stresses are matched to obtain material parameters. These stresses are calculated as explained in section 2.2 however some definitions can be varied as shown below. An overview of the variations is given in Tabel 4 .

\subsubsection{Deformation gradient calculation}

The deformation gradient $\boldsymbol{F}$ is used in both the model and the experimental stress calculation. In an actual planar planar biaxial experiment, $\boldsymbol{F}$ can be calculated using different input data and applying different assumptions.

Input data. Different input data can be used to calculate the deformation tensor $\boldsymbol{F}$, each representing the actual strain field with different accuracy. A deformation tensor based on the displacements of the gripping mechanism is the least 
accurate, since gaps, tear or slippage of the specimen at the gripping mechanism can occur, and since the complete area between the gripping mechanisms is assumed to be homogeneous. Two other possibilities measure the strain optically by means of a camera. Strain mapping represents the strain field the most accurately, with accuracy increasing as the resolution of the mapping increases. However, natural or artificial texture for strain mapping can be difficult to obtain, since the specimen is completely immersed during testing and because natural texture is usually not very pronounced in biological samples. The third possibility is to calculate the deformation tensor using markers and marker tracking. This possibility assumes the strain field between the markers to be homogeneous and hence has an intermediate accuracy. These three methods that are used in experimental practice to determine the deformation gradient were used to calculate the model stresses.

Incorporation or neglection of shear terms. The stress state created in the tissue when under tension depends on the testing set-up that is used, and the orientation of the fibre families in the sample. In a planar biaxial set-up using rakes, it is sometimes assumed that the amount of shear is limited and can be neglected [6, 21, 22. In this case the deformation gradient consists of diagonal terms only, which simplifies the stress calculations. To verify whether this assumption is valid, parameter fitting was performed using a deformation gradient that either neglects shear or takes shear into account.

For this case, a second FE model was built that models the full sample and the rakes. Hence, the symmetry assumption is not made in this FE model. The dimensions of the model are shown in Figure 6. The specimen is modelled using 64000 S4R elements and with material properties of GOH-parameter set 1 (see Table 2). Each rake is modelled using 10 slender beam elements (B33) with material properties of stainless steel: a Young's modulus of 195GPa and a Poisson ratio of 0.31 . The rakes are tied to the rakes' attachment sites of the sample. Boundary conditions are as follows: at each sample edge displacements at the 
outer end of the rakes are applied in the length direction of the rakes and prohibited in the perpendicular direction. The center of the sample is fixed in both directions. Four virtual markers are defined in a square around the sample center, spaced $1.8 \mathrm{~mm}$ apart. Data is again exported as in an actual planar biaxial experiment: forces in the longitudinal direction of the rakes and displacements of the markers are tracked. Lateral forces can typically not be measured in a planar biaxial experiment using rakes and therefore are not exported.

The deformation gradient is calculated from the marker displacements. When shear is taken into account, the deformation gradient can be calculated using the method described by Hoffman and Grigg [23] to measure strains in soft tissues. This finite element based method treats the markers on the tissue as nodes of a four node isoparametric element and uses finite element mathematics to calculate the strain tensor at the center of mass of the element:

$$
\boldsymbol{F}_{W S}=\left[\begin{array}{ccc}
F_{11} & F_{12} & 0 \\
F_{21} & F_{22} & 0 \\
0 & 0 & F_{33}
\end{array}\right]
$$

When shear is neglected, the stretch ratios $\lambda_{i}$ on the diagonal of the deformation gradient are calculated from the length changes in the $i-t h$ direction:

$$
\boldsymbol{F}_{N S}=\left[\begin{array}{ccc}
\frac{l_{x}}{l_{x, \text { initial }}} & 0 & 0 \\
0 & \frac{l_{y}}{l_{y, \text { initial }}} & 0 \\
0 & 0 & F_{33}
\end{array}\right]
$$

The strain in the third direction $F_{33}$ is always calculated from the incompressibility constraint: $\operatorname{det}(\boldsymbol{F})=1$. A parameter fitting was conducted using both deformation gradients. In the simulations of a quarter sample, the deformation gradient is calculated using equation 14 .

\subsubsection{Initial section width $l_{i 0}$}

Section 2.2 explains that the experimental stress is calculated as force divided by an undeformed section surface $A_{i, 0} . A_{i, 0}$ is defined by the initial specimen 
thickness $h_{0}$ and the initial section width $l_{i 0}$ in the i-th direction. The accepted definition of the section width $l_{i 0}$ is unclear from literature. $l_{i 0}$ can be either the initial specimen length $L_{s i, 0}$, the initial length covered by the rakes $L_{r i, 0}$ or the initial rakes' width $R_{w i, 0}$ (see Figure 5). The experimental stress is calculated with each of these three definitions.

\subsection{Variations to the parameter fitting procedure}

\subsubsection{Classic parameter fitting}

The classic parameter fitting procedure is described in section 2.2 In the objective function (Equation 1), the model stresses are altered until they match the experimental stresses as good as possible. The result of this optimization process are the material parameters of the constitutive model. This is done in Matlab R2013a using the non-linear least squares (lsqnonlin) routine and the trust-region-reflective optimization algorithm.

\subsubsection{Inhomogeneity from Experimental set-up Corrected (IEC) parameter fit- ting}

A new procedure, the IEC-parameter fitting, is presented in Figure 7 In this procedure, a FE simulation of the biaxial experiment with material parameters obtained from the classic procedure is performed. Model and experimental stresses of this FE simulation are known and a correction vector can be determined. In contrast to the correction factor defined earlier, which was a scalar determined in an optimization process, the correction vector $g(i)$ is calculated for each time point $i$ :

$$
g_{j}(i)=\frac{\sigma_{m o d, j}^{F E}(i)}{\sigma_{e x p, j}^{F E}(i)} \text { with } j=x, y
$$

These correction vectors can then be combined with the original stress data in a new parameter fitting round. This process of conducting a FE simulation, determining correction vectors and parameter fitting with these vectors, can be repeated until a stop criterion is fulfilled. An example of such a stop criterion is that the RMSE between the correction vector $g$ and an all-ones vector are 
below a specific threshold.

An overview of all variations in the FE simulations and the data processing is given in Table 4. Values that are not reported explicitly in each simulation are set to the default values (boldfaced in the table).

\subsection{Consequences of suboptimal material parameters}

The importance of determining the correct material parameters is exposed when these parameters are used in subsequent FE simulations. In this paragraph, the consequences of the different variations described above are demonstrated with a third series of FE simulations. In these simulations a human abdominal aortic artery is modelled as a cylindrical tube using Abaqus/Standard 6.12-2. The mesh contains $30000 \mathrm{C} 3 \mathrm{D} 8 \mathrm{H}$ elements. The geometric values are based on [18, 24] and [25]. Due to symmetry, only the half of the cylinder needs to be modelled. At the start, the artery has a stress-free geometry with an opening angle $\Theta_{0}=120^{\circ}$, a wall thickness of $2.69 \mathrm{~mm}$, a length $L=10 \mathrm{~mm}$ and an outer radius $R_{o}=12 \mathrm{~mm}$ (see Figure 8).

The boundary conditions assure that the bottom segment is in the 12-plane and the cylinder length is along the 3-axis. Loading is applied in consecutive steps as shown in Figure 9. First, the segment is closed to form a cylinder, then an axial prestretch of $\lambda_{33}=1.08$ is applied and finally the cylinder is pressurized with a systolic pressure of $P_{\text {sys }}=126 \mathrm{mmHg}$.

This simulation is conducted with different GOH-material parameter sets, each one obtained through parameter fitting. Different datasets from the above planar biaxial simulation, different definitions and different fitting procedures are used (see Table 5). For each parameter set the minimal and maximal stresses are compared.

\section{Results}

This section presents the results of each of the variations in different subsections: first variations to the experimental set-up, secondly variations to the 
stress calculations and then variations to the parameter fitting approach. The final subsection shows the results of the examination of the consequences of suboptimal material parameters.

\subsection{Variations to the experimental set-up}

The variations to the experimental set-up are subdivided into three aspects: sample geometry, loading protocol and material. For each of these variations the two quality measures, i.e. the correction factor $f$ and the inhomogeneity $h$, are shown. Optimal planar biaxial testing conditions show a good correspondence between model and experimental stress (i.e. $f$ close to 1 ) and create a homogeneous stress field (i.e. $h$ close to 0 ).

\subsubsection{Geometry}

Number of rakes $\mathfrak{G}$ marker position. For four or more rakes $f$ is more or less constant and close to the ideal value of 1 for every marker position $L_{m} / L_{r}$ (Figure 10. left). With an increasing number of rakes, the stress field is more homogeneous and the inhomogeneity slopes are smaller (Figure 10 right). As expected, the inhomogeneity $h$ increases linearly as the marker position approaches the rakes' attachment sites, but the incremental advantage decreases as more rakes are used.

Rakes' width $R_{w}$. Both the correction factor and the inhomogeneity improve as the rakes cover a larger section of the specimen edge (Figure 11). With a small rakes' width $R_{w} / L_{r}$, the applied load is concentrated in a central section of the edge. Increasing the inter-rake distance, increases $R_{w} / L_{r}$. Hence the load is applied on a larger section of the edge, resulting in a more homogeneous stress-strain field and a better correspondence between model and experimental stresses.

Apron size $\left(L_{s}-L_{r}\right)$. The apron size has no significant effect on either the correction factor $f$ or the inhomogeneity $h$ (Figure 12). The size of the apron decreases as the rake-to-specimen surface ratio $L_{r} / L_{s}$ increases. Since the scale 
of both $f$ and $h$ is small and no clear trend can be observed for either quality measure as a function of apron size, it can be concluded that for different apron sizes they remain relatively constant.

\subsubsection{Loading protocol}

Ratios 85 strain levels. The correction factor $f$ and the inhomogeneity $h$ are both dependent on the strain level and on the ratio of the testing protocol (Figure 13). For the custom ratio, $f$ remains approximately constant, but for the other ratios, $f$ improves with an increasing strain. For the $0.5: 1$ ratio the correction factor $f$ is closest to 1 for each strain level. In contrast, the $1: 0.5$ ratio provides the most homogeneous stress field for this type of material and specimen orientation. The inhomogeneity $h$ also increases with the strain.

\subsubsection{Material}

Stiffness $\&$ constitutive model. For the same specimen geometry and loading protocol a different material parameter set leads to a different correction factor $f$ and a different inhomogeneity $h$ for every strain level (Figure 14. Moreover, when comparing GOH-1 and NH, which are different constitutive models with the same linear stiffness, it can be observed that they create a similar inhomogeneity $h$, but show no correspondence in the correction factor $f$.

Fibre angle. No clear trend can be observed for either quality measure. Hence, it can be concluded that the fibre angle $\alpha$ has no significant effect for this type of material and geometry (Figure 15).

\subsection{Variations to the stress calculations}

\subsubsection{Deformation gradient}

Input data. Different input data is used to calculate the deformation gradient

tensor $\left(\boldsymbol{F}_{\text {rakes }}, \boldsymbol{F}_{\text {markers }}\right.$ and $\boldsymbol{F}_{\text {strainmap }}$ ) lead to different model stresses (Figure 16). As expected the model stresses with a deformation gradient tensor based on markers and strain map correspond well for these testing conditions and type of material. 
Incorporation or neglection of shear terms. Table 6 shows the parameters obtained through parameter fitting either neglecting shear (NS) or taking shear into account (WS). The Mean Percentage Error (MPE) is calculated between the input parameters and the fitted parameters. Both parameter sets and the MPE are similar.

\subsubsection{Initial section width $l_{i 0}$}

Different definitions of the section width $\left(R_{w}, L_{r}\right.$ and $\left.L_{s}\right)$ lead to different experimental stresses (Figure 16). The experimental stress calculated with $L_{r}$ as section width, corresponds best with the model stresses.

\subsection{Variations to the parameter fitting procedure}

The parameters obtained with the IEC-parameter fitting procedure correspond better to the input parameters than those obtained with the classic procedure (Table 7). The fitting was performed on the data from the 1:1 ratio and on the data from three ratios $(1: 1,0.5: 1,1,0.5)$ combined. The RMSE is calculated between the stresses from the input parameters and the stresses from the parameters obtained through fitting. The MPE is calculated between the input parameters and the fitted parameters. Note that RMSE and MPE were chosen

to assess the goodness of fit, since the Pearson correlation coefficients and the coefficients of determination $R^{2}$ all resulted 0.99. However, these goodness of fit values must be handled with care: for example, the RMSE is very sensitive to the maximum stress values in the dataset. Since these values are different with the 0.5:1 and 1:0.5 ratio, the RMSE should not be used to compare between a single ratio and multiple ratios.

The RMSE and MPE both decrease in the IEC-parameter fitting with respect to the classic one. After the second iteration of the IEC-parameter fitting, the material parameters approximate the input parameters very precisely. The MPE does not show a clear trend when using one ratio or multiple ratios. 


\subsection{Consequences of suboptimal material parameters}

A second series of FE simulations is conducted to investigate the consequences of using suboptimal material parameters. The parameter sets used for these FE simulations are shown in Table 8 . These parameter sets are obtained through fitting with different datasets, different definitions of section width and different fitting procedures (see Table 5).

Table 9 shows the minimal and maximal stresses and strains of one element at systolic pressure. This element is located at the inner wall in the middle of the cylindrical segment (see Figure 8). Only the Cauchy stresses $\sigma_{\text {circ }} \& \sigma_{a x}$ and the stretch ratios $\lambda_{\text {rad }} \& \lambda_{\text {circ }}$ are presented, since $\sigma_{\text {rad }}$ and $\lambda_{a x}$ are subjected to boundary conditions and hence are equal in every simulation. Figure 17 shows the stress (Cauchy stresses $\sigma_{\text {circ }} \& \sigma_{a x}$ ) and strain (logarithmic strains $\ln \left(\lambda_{\text {rad }}\right)$ $\left.\& \ln \left(\lambda_{\text {circ }}\right)\right)$ fields for each simulation.

With respect to the ground truth, the largest deviation in stresses and strains occurs for the simulations SW1, SW3 and SL2 (Table 9 \& Figure 17). The stresses and strains are overestimated up to three times and underestimated up to half of the ground truth value. Comparing the different strain levels (SL2, SL3 and SL4) with the ground truth, SL3 shows the smallest deviation in both stresses and strains. The large deviation of SL2 is due to an inadequate data set (i.e. a too small strain level) for parameter fitting in order to fully characterize the non-linear behaviour. The deviations of SL4 are related to the amount of inhomogeneities in the stress-strain field that increase with increasing strain. For this type of material and geometry SL3 has the best balance between high enough strains (for full material characterization), but not too high (to avoid large inhomogeneities). These aspects are also reflected in the parameter sets (see Table 8). As to the definition of the section width, using $R_{w}$ (SW1) overestimates the stresses, while using $L_{s}$ (SW3) underestimates the strains, both with respect to the ground truth and to using $L_{r}$ as section width (SL4). The stresses and strains from RM, where the material parameters are obtained via parameter fitting on multiple ratios, are comparable with those from SL4, 
where only one ratio was used for parameter fitting. Finally, the stresses and strains of the simulation with the material parameters obtained via the new IECparameter fitting procedure (SL2-IEC, SL3-IEC and SL4-IEC) approximate the ground truth stresses and strains best.

\section{Discussion}

The goal of this study was to determine optimal testing conditions for rakebased planar biaxial testing of soft biological tissue and to bring consensus on the calculation method for model and experimental stresses. Apart from examining the consequences of the use of suboptimal parameters in FE studies, also a new and efficient parameter fitting procedure was introduced.

\subsection{Variations to the experimental set-up}

To determine optimal testing conditions, variations to the experimental setup were investigated in a series of FE simulations: number of rakes, marker position, rakes' width, apron size, ratio, strain level, stiffness, constitutive model and fibre angle. Better testing conditions show a higher correspondence between experimental and model stresses (i.e. $f \approx 1$ ) and create a more homogeneous stress field (i.e. $h \approx 0$ ).

The results show that better testing conditions are obtained with an increasing number of rakes and a more central marker position. However, the signal-to-noise ratio of the strain measurement decreases for a more central marker position. The incremental advantage of increasing the number of rakes decreases, hence five rakes can be defined as a practical optimum: with a relatively constant correction factor $f$ and a small inhomogeneity slope, a central marker position becomes less crucial (Figure 10. Increasing the rakes' width, such that the load is applied at a large segment of the specimen edge also results in improved testing conditions (Figure 11). Accordingly, inter-rake distance should be as wide as practically possible, which brings adjacent corner rakes close to each other. The above results make sense as increasing the number 
of discrete loading points and spreading the loading points along the complete specimen side improves the approximation of a continuous load distribution. Moreover, central markers experience less effects of the boundary conditions (i.e. the discrete load application). These results are in correspondence with the findings in $[9]$.

The size of the apron, i.e. the specimen surface outside of the rakes' surface, and the fibre angle $\alpha$ have no significant effect on the correction factor $f$ and inhomogeneity $h$ for this type of material and geometry (Figures $12 \& 15$. However, this must be considered with nuance: with a higher fibre stiffness (i.e. a higher $k_{1}$ in the GOH-model), the influence of $\alpha$ might be more significant. Additionally, a very small apron will result in a more inhomogeneous stressstrain field as excessive deformation occurs at the rakes. However, as this will result in early material failure, a very small apron is not used in practice.

On the contrary, the correction factor $f$ and inhomogeneity $h$ do depend on the loading ratio, strain level, constitutive model and material stiffness, but do not show a clear trend (Figures $13 \&$ 14). This means that no correction factor can be determined for a specific testing condition, since it depends on the material that is tested. This supports the idea of the new IEC-fitting procedure in which correction vectors are determined based on a FE simulation of the planar biaxial experiment with a first approximate material parameter set obtained via the classic fitting procedure.

Eilaghi et al. 9] found that non-uniformity in the placement or spacing of rakes disrupts the homogeneity of the stress and strain field. In practice, rakes' spacing easily deforms slightly when used. Therefore, it would also be recommended to use the IEC-parameter fitting procedure to overcome the inhomogeneity in the stress-strain field caused by irregularities in rakes' spacing. Eilaghi et al. 9] also investigated the effect of a changing shape of the apron and concluded that neither the apron width nor the apron shape is a critical determinant of the interior strain field. 


\subsection{Variations to the stress calculations}

The model and experimental stresses differ according to the input data used for the deformation gradient $\boldsymbol{F}$ and the definition of the initial section width $l_{i 0}$ (Figure 16). The deformation gradient based on the rakes overestimates the model stresses, due to the gaps that originate between the tissue and the rakes when the load is applied. On the other hand, the deformation gradient

based on a strain map or based on markers correspond well provided the testing conditions as explained above. Poorer testing conditions will cause a more inhomogeneous stress-strain field. It is possible that in this case strain map shows better results than markers. Nevertheless, in these simulations the material to be tested is homogeneous, while the choice between strain mapping and markers should also be influenced by the homogeneity of the tissue. For strongly inhomogeneous tissue such as atherosclerotic tissue, different regions need to be characterized separately and strain mapping allows easier identification of smaller homogeneous regions.

As to the definition of the section width, the length covered by the rakes $L_{r}$ should be used as was also recently mentioned by [11. In this case, the experimental stresses match the model stresses best (Figure 16. In contrast, the experimental stress based on the rakes' width $R_{w}$ overestimates the model stress and the experimental stress based on the sample width $L_{s}$ underestimates the model stress. This is caused by the under- and overestimation of the section surface, respectively. These findings are not in agreement with Eilaghi et al. [9], who propose a correction for the stress calculation by taking $35 \%$ of the apron width into account. An explanation for them finding a constant fraction of $35 \%$ can be that while $L_{r}$ and $L_{s}$ were varied, the rake-to-specimen surface ratio $L_{r} / L_{s}$ was approximately constant at 0.9 .

The model and experimental stresses calculated with a deformation gradient that incorporates shear are similar to those calculated with a deformation gradient neglecting shear. The result of the parameter fitting confirms this as there is no significant difference in the fitted parameters according to the MPE (see Table 6). One important nuance is the fact that this FE simulation was 
conducted for a symmetric material, which is a material that is symmetric with respect to the test axes. The negligible difference in the model and experimental stresses between a deformation gradient $\boldsymbol{F}$ with shear and without shear can be explained by the fact that for symmetric materials no shear stress will be induced because the lateral forces on each of the rakes will cancel out. Of course this is only true when the rakes are regularly placed with a uniform space in between.

Shear stress will occur when a material is asymmetric with respect to the test axes. Hence, in a rake-based set-up the resultant lateral force on the rakes will not be zero and a shear stress will be induced. However, in most planar biaxial set-ups, only normal (longitudinal) forces are measured. Typically, shear (lateral) forces cannot be measured, which complicates the handling of shear in planar biaxial testing. The general handling of shear in a rake-based planar biaxial tests (e.g. in asymmetric materials) is beyond the scope of this paper, but will be considered as a subject for future work. Based on the current results, a clear recommendation is to align the symmetry axes of the specimen as good as possible with the test axes and to exert caution with test results when the symmetry axes are not or not accurately known.

\subsection{Variations to the parameter fitting procedure}

A new IEC-fitting procedure is proposed next to the classic procedure. The IEC-method accounts for the error resulting from an incorrect section surface estimation and an inhomogeneous stress-strain field. The results indicate that, even in perfect circumstances (i.e. when noise introduced by practical inaccuracies and sensors is absent) the correct material parameters cannot be found with the classic fitting procedure. On the contrary, the improvements of the IEC-parameter fitting procedure are already significant after the first iteration. After the second iteration, the IEC-fitting procedure is able to approximate the correct material parameters very precisely (Table 7). Accordingly, the decrease of the RMSE and the MPE is significant when using the IEC-fitting procedure compared to the classic fitting procedure. Combining the data of different ratios 
for fitting does not always result in improved parameters as there is no clear trend in the MPE. However, using multiple ratios is still beneficial in an actual experiment as in reality the model cannot perfectly catch the experimental data. The IEC-fitting procedure has now been tested on simulated data and the improvement is significant. Therefore, we suggest the method to be applied to actual experimental data.

Inverse finite element (IFE) modelling is another method that can be used for parameter fitting. However, IFE is more computationally intensive than the classic and the IEC-procedure. Consider a fitting problem that requires $n$ iterations to solve the non-linear optimization using the classic fitting procedure. For the same problem, IFE would also need approximately $n$ iterations and hence $n$ FE simulations to solve. The IEC-fitting procedure on the other hand would need only $k \mathrm{FE}$ simulations with $k<<n$, combined with $k * n$ iterations to solve. Moreover, a multistart function should be used to ensure that a global minimum is found, by which the total number of iterations increases even further. Hence, the benefit of the IEC-fitting procedure compared to IFE is the computational efficiency. With respect to the classic fitting procedure, the IEC-parameter fitting procedure takes the inhomogeneity caused by the experimental set-up and a correction for the section surface into account. Nevertheless, highly inhomogeneous material should still be characterized with IFE combined with strain mapping.

\subsection{Consequences of suboptimal material parameters}

In the other series of FE simulations to investigate the consequences of suboptimal material parameters, the geometry and loading \& boundary conditions are the same, only the material parameters differ (Table 8). Each parameter set is obtained via parameter fitting, but using different datasets, different definitions of section width and different fitting procedures. The deviations in stresses and strains are smallest when the IEC-fitting procedure is used (Table 9 \& Figure 17). In general the largest deviations over- and underestimate the stresses and strains up to three times and respectively a half of the ground truth value. 
They are dependent on the strain level of the protocol and on the definition of section width $l_{i 0}$, but independent of the use of a single or multiple ratios. This independence is in correspondence with the finding that multiple ratios do not result in improved parameters. Comparing SL2, SL3 and SL4 reflects the importance of applying high enough strains for full material characterization, but not too high strains to avoid high stress-strain field inhomogeneities. However, the 'ideal' strain range is material dependent, which makes it impossible to define one range for a specific testing condition. The inhomogeneities are accounted for when using the IEC-fitting method: the performance of SL3-IEC and SL4IEC is almost equally good. To conclude, the newly proposed IEC-parameter fitting procedure leads to stresses and strains approximating the ground truth quite precisely.

\subsection{General discussion}

Besides planar biaxial tests, other testing methods can be used for material characterization of soft biological tissues such as extension-inflation tests, e.g. [26] or uniaxial tests, e.g. [27, 28]. Depending on the boundary conditions of the set-up, its influence on the results of the parameter fitting can be more or less pronounced. Therefore, we recommend to investigate the parameter fitting results for these other testing methods. The same advice is given for planar biaxial testing with other boundary conditions, such as clamps or sutures. The IEC-parameter fitting method can be extended to other testing methods and is expected to lead to improved parameter fitting.

There are many material descriptions available for fibrous tissue architectures, where very often an additive decomposition is made between the energy contribution of an isotropic matrix and an anisotropic fibrous constituent. For this anisotropic constituent, the angular integration method is considered the exact method to model the contribution of distributed fibers (see [29, 30]), but computationally intensive. On the other hand, generalized structure tensors are used to overcome this computational complexity, be it at the cost of inducing averaging errors [31, 32]. The GOH-model used in this manuscript is an example 
of the latter. Indeed, it does suffer from these averaging errors, but two reasons have led us to using this formulation: ease of numerical implementation which is why it is also readily available in common FE software packages and, consequently, high prevalence in the research community. More accurate material descriptions exist (e.g. [33, 30]), but the qualitative results and consequently the message of this manuscript are not influenced by the averaging errors of the current approach.

As the influences of different aspects on the quality of the parameter fitting and the effects of suboptimal material parameters are significant, the importance of complete reporting of biaxial experiments is stressed. Only this will allow proper comparison of material parameters from different studies in the future. An approach that should also be considered is the use of open access databases for the reporting of biaxial experiments, in the spirit of the Virtual Physiological Human project (http://www.vph-institute.org). Furthermore, this allows to perform a parameter fitting of a different constitutive model on the data. Likewise, the data should be well documented.

\section{Conclusion}

Recommendations for best practice in rake-based planar biaxial testing and subsequent parameter fitting are formulated based on a series of FE simulations with varying testing conditions, definitions and parameter fitting procedures. Regarding the latter, a new IEC-parameter fitting procedure is proposed that accounts for the error resulting from an incorrect section surface and from an inhomogeneous stress and strain field caused by the boundary conditions of the experimental set-up, while being far more computationally efficient than an inverse FE fitting approach.

Guidelines for reporting of planar biaxial testing are formulated as the results are strongly influenced by choices made in the experimental set-up and in the data analysis. They affect the homogeneity of the stress and strain field (e.g. number of rakes, Figure 10 and the model and experimental stress calculation 
(e.g. definition section surface, Figure 16). Adopting these recommendations and guidelines, summarized in the appendix, will allow proper comparison of material parameters from different studies in the future.

\section{Acknowledgements}

This work was supported by a postdoctoral fellowship of the Research FoundationFlanders (FWO), a research project of FWO (G093211N), a research project of KU Leuven (IDO 09/012) and the Materialise chair for patient-specific, imagebased biomechanics.

\section{Appendix}

Summary of the recommendations for planar biaxial testing

- experimental set-up

- minimum five rakes

- inter-rake distance as large as possible and the corner rakes as close to each other as practically possible

- avoid irregularities in spacing or positioning of rakes

- central marker position (within central $25 \%$ of sample according to [5])

- data analysis

- use the length covered by the rakes $L_{r}$ as section width $l_{i 0}$ for the experimental stress calculation

- calculate deformation gradient based on markers or on strain mapping

- shear terms in the deformation gradient can be neglected in the case of symmetric rake positions and symmetric material (i.e. symmetry with respect to the test axes) 
- account for inhomogeneities by using the IFE fitting approach or, for increased efficiency, the newly proposed IEC approach.

Summary of the guidelines for reporting of planar biaxial experiments

- specify the following details of the experimental set-up:

- number of rakes

- length covered by the rakes $L_{r}$

- rakes' width $R_{w}$

- marker positions

- loading protocol (ratio's and marker strains)

- possible irregularities in rakes positions and spacing

- specify the following details on data analysis

- deformation gradient calculation (input data and shear handling)

- section width used for experimental stress calculation

- parameter fitting procedure approach

\section{References}

[1] F. Migliavacca, L. Petrini, M. Colombo, F. Auricchio, R. Pietrabissa, Mechanical behavior of coronary stents investigated through the finite element method, Journal of Biomechanics 35 (6) (2002) 803-811. doi:http://dx.doi.org/10.1016/S0021-9290(02)00033-7. URL http://www.sciencedirect.com/science/article/pii/ S0021929002000337

[2] C. Conway, F. Sharif, J. P. McGarry, P. E. McHugh, A Computational TestBed to Assess Coronary Stent Implantation Mechanics Using a PopulationSpecific Approach, Cardiovascular Engineering and Technology 3 (4) (2012) 374-387. doi:10.1007/s13239-012-0104-8

URL http://dx.doi.org/10.1007/s13239-012-0104-8 
[3] Y. C. Fung, Biomechanics Mechanical properties of Living Tissues, Springer-Verlag New York Inc, 1981.

[4] J. D. Humphrey, Cardiovascular solid mechanics: Cells, tissues and organs, Springer, 2002.

[5] M. S. Sacks, Biaxial Mechanical Evaluation of Planar Biological Materials, Journal of elasticity and the physical science of solids 61 (1-3) (2000) 199246. doi:10.1023/A:1010917028671.

URL http://dx.doi.org/10.1023/A\{\%\}3A1010917028671

[6] G. Sommer, A. Schriefl, G. Zeindlinger, A. Katzensteiner, H. Aindhofer, A. Saxena, G. A. Holzapfel, Multiaxial mechanical response and constitutive modeling of esophageal tissues: Impact on esophageal tissue engineering, Acta Biomaterialia 9 (12) (2013) 9379-9391. doi:http://dx.doi.org/10.1016/j.actbio.2013.07.041.

URL http://www.sciencedirect.com/science/article/pii/ S1742706113003899

[7] W. Sun, M. S. Sacks, M. S. Scott, Effects of Boundary Conditions on the Estimation of the Planar Biaxial Mechanical Properties of Soft Tissues, Journal of Biomechanical Engineering 127 (4) (2005) 709-715.

[8] D. R. Nolan, J. P. McGarry, On the correct interpretation of measured force and calculation of material stress in biaxial tests., Journal of the mechanical behavior of biomedical materials 53 (2016) 187-99. doi:10.1016/j.jmbbm.2015.08.019

URL http://www.sciencedirect.com/science/article/pii/ S1751616115002908

[9] A. Eilaghi, J. G. Flanagan, B. G. Wayne, E. C. Ross, Strain Uniformity in Biaxial Specimens is Highly Sensitive to Attachment Details, Journal of Biomechanical Engineering 131 (2009) 91003. 
[10] S. D. Waldman, J. Michael Lee, Boundary conditions during biaxial testing of planar connective tissues. Part 1: Dynamic Behavior, Journal of Materials Science: Materials in Medicine 13 (10) (2002) 933-938. doi: 10.1023/A:1019896210320.

URL http://dx.doi.org/10.1023/A\{\%\}3A1019896210320

[11] W. Zhang, Y. Feng, C.-H. Lee, K. L. Billiar, M. S. Sacks, A Generalized Method for the Analysis of Planar Biaxial Mechanical Data Using Tethered Testing Configurations, Journal of Biomechanical Engineering 137 (6) (2015) 064501. doi:10.1115/1.4029266.

URL http://biomechanical.asmedigitalcollection.asme.org/ article.aspx?doi=10.1115/1.4029266

[12] R. W. Ogden, G. Saccomandi, I. Sgura, Fitting hyperelastic models to experimental data, Computational Mechanics 34 (6) (2004) 484-502. doi: 10.1007/s00466-004-0593-y

URL http://dx.doi.org/10.1007/s00466-004-0593-y

[13] M. S. Sacks, W. Sun, Multiaxial mechanical behavior of biological materials, Annual Review of Biomedical Engineering 5 (1) (2003) 251-284. doi:10.1146/annurev.bioeng.5.011303.120714

URL http://dx.doi.org/10.1146/annurev.bioeng.5.011303.120714

[14] N. T. Jacobs, D. H. Cortes, E. J. Vresilovic, D. M. Elliot, Biaxial Tension of Fibrous Tissue: Using Finite Element Methods to Address Experimental Challenges Arising From Boundary Conditions and Anisotropy, Journal of Biomechanical Engineering 135 (2) (2013) 21004-21010.

[15] P. Kurowski, Avoiding pitfalls in FEA, Machine Design 66 (1994) 78-86.

[16] J. Bonet, R. D. Wood, Nonlinear Continuum Mechanics for Finite Element Analysis, Cambridge University Press, 1997.

[17] G. A. Holzapfel, Nonlinear Solid Mechanics: A Continuum Approach for Engineering, John Wiley \& Sons, 2000. 
[18] B. Sonesson, F. Hansen, T. Länne, Compliance and diameter in the human abdominal aorta - influence of age and sex, European Journal of Vascular Surgery 7 (1993) 690-697.

[19] L. Horny, T. Adamek, H. Chlup, R. Zitny, Age estimation based on a combined arteriosclerotic index, International Journal of Legal Medicine 126 (2) (2012) 321-326. doi:10.1007/s00414-011-0653-7.

URL http://dx.doi.org/10.1007/s00414-011-0653-7

[20] T. C. Gasser, R. W. Ogden, G. A. Holzapfel, Hyperelastic modelling of arterial layers with distributed collagen fibre orientations, Journal of the Royal Society Interface 3 (2006) 15-35.

[21] S. a. O'Leary, E. G. Kavanagh, P. a. Grace, T. M. McGloughlin, B. J. Doyle, The biaxial mechanical behaviour of abdominal aortic aneurysm intraluminal thrombus: Classification of morphology and the determination of layer and region specific properties, Journal of Biomechanics 47 (6) (2014) 1430-1437. doi:10.1016/j.jbiomech.2014.01.041.

[22] J. P. Vande Geest, M. S. Sacks, D. a. Vorp, A planar biaxial constitutive relation for the luminal layer of intra-luminal thrombus in abdominal aortic aneurysms, Journal of Biomechanics 39 (13) (2006) 2347-2354. doi:10. $1016 / \mathrm{j} \cdot \mathrm{jbiomech} .2006 .05 .011$.

[23] A. H. Hoffman, P. Grigg, A method for measuring strains in soft tissue Journal of Biomechanics 17 (10) (1984) 795-800. doi:http://dx.doi.org/10.1016/0021-9290(84)90110-6

URL http://www.sciencedirect.com/science/article/pii/ 0021929084901106

[24] H. C. Han, Y. C. Fung, Direct measurement of transverse residual strains in aorta, American Journal of Physiology - Heart and Circulatory Physiology 270 (2) (1996) H750--H759. 
[25] H. Weisbecker, D. M. Pierce, P. Regitnig, G. A. Holzapfel, Layer-specific damage experiments and modeling of human thoracic and abdominal aortas with non-atherosclerotic intimal thickening, Journal of the Mechanical Behavior of Biomedical Materials 12 (0) (2012) 93-106. doi:http://dx.doi.org/10.1016/j.jmbbm.2012.03.012

URL http://www.sciencedirect.com/science/article/pii/ S1751616112000926

[26] N. Famaey, G. Sommer, J. V. Sloten, G. A. Holzapfel, Arterial clamping: Finite element simulation and in vivo validation, Journal of the Mechanical Behavior of Biomedical Materials 12 (0) (2012) 107-118. doi:http://dx.doi.org/10.1016/j.jmbbm.2012.03.010

URL http://www.sciencedirect.com/science/article/pii/ S1751616112000902

[27] D. M. Pierce, F. Maier, H. Weisbecker, C. Viertler, P. Verbrugghe, N. Famaey, I. Fourneau, P. Herijgers, G. A. Holzapfel, Human thoracic and abdominal aortic aneurysmal tissues: Damage experiments, statistical analysis and constitutive modeling, Journal of the Mechanical Behavior of Biomedical Materials 41 (0) (2015) 92-107. doi:http://dx.doi.org/10.1016/j.jmbbm.2014.10.003

URL http://www.sciencedirect.com/science/article/pii/ S1751616114003166

[28] G. A. Holzapfel, Determination of material models for arterial walls from uniaxial extension tests and histological structure, Journal of Theoretical Biology 238 (2) (2006) 290-302. doi:http://dx.doi.org/10.1016/j.jtbi.2005.05.006

URL http://www.sciencedirect.com/science/article/pii/ S0022519305002080

[29] Y. Lanir, Constitutive equations for fibrous connective tissues., Journal of biomechanics 16 (1) (1983) 1-12. doi:10.1016/0021-9290(83)90041-6. 
[30] D. H. Cortes, D. M. Elliott, Accurate prediction of stress in fibers with distributed orientations using generalized high-order structure tensors, Mechanics of Materials 75 (2014) 73-83. doi:10.1016/j.mechmat.2014.04. 006.

URL http://dx.doi.org/10.1016/j.mechmat.2014.04.006

[31] S. Federico, W. Herzog, Towards an analytical model of soft biological tissues, Journal of Biomechanics 41 (16) (2008) 3309-3313. arXiv: 56549110892, doi:10.1016/j.jbiomech.2008.05.039.

[32] D. H. Cortes, S. P. Lake, J. A. Kadlowec, L. J. Soslowsky, D. M. Elliott, Characterizing the mechanical contribution of fiber angular distribution in connective tissue: Comparison of two modeling approaches, Biomechanics and Modeling in Mechanobiology 9 (2010) 651-658. doi: $10.1007 / \mathrm{s} 10237-010-0194-\mathrm{x}$.

[33] R. Fan, M. S. Sacks, Simulation of planar soft tissues using a structural constitutive model: Finite element implementation and validation, Journal of Biomechanics 47 (9) (2014) 2043-2054. doi:10.1016/j.jbiomech. 2014.03 .014

URL http://dx.doi.org/10.1016/j.jbiomech.2014.03.014

[34] M. Smoljkić, J. Vander Sloten, P. Segers, N. Famaey, Non-invasive, energybased assessment of patient-specific material properties of arterial tissue, Biomechanics and Modeling in Mechanobiology (2015) 1-12doi:10.1007/ s10237-015-0653-5.

URL http://dx.doi.org/10.1007/s10237-015-0653-5 


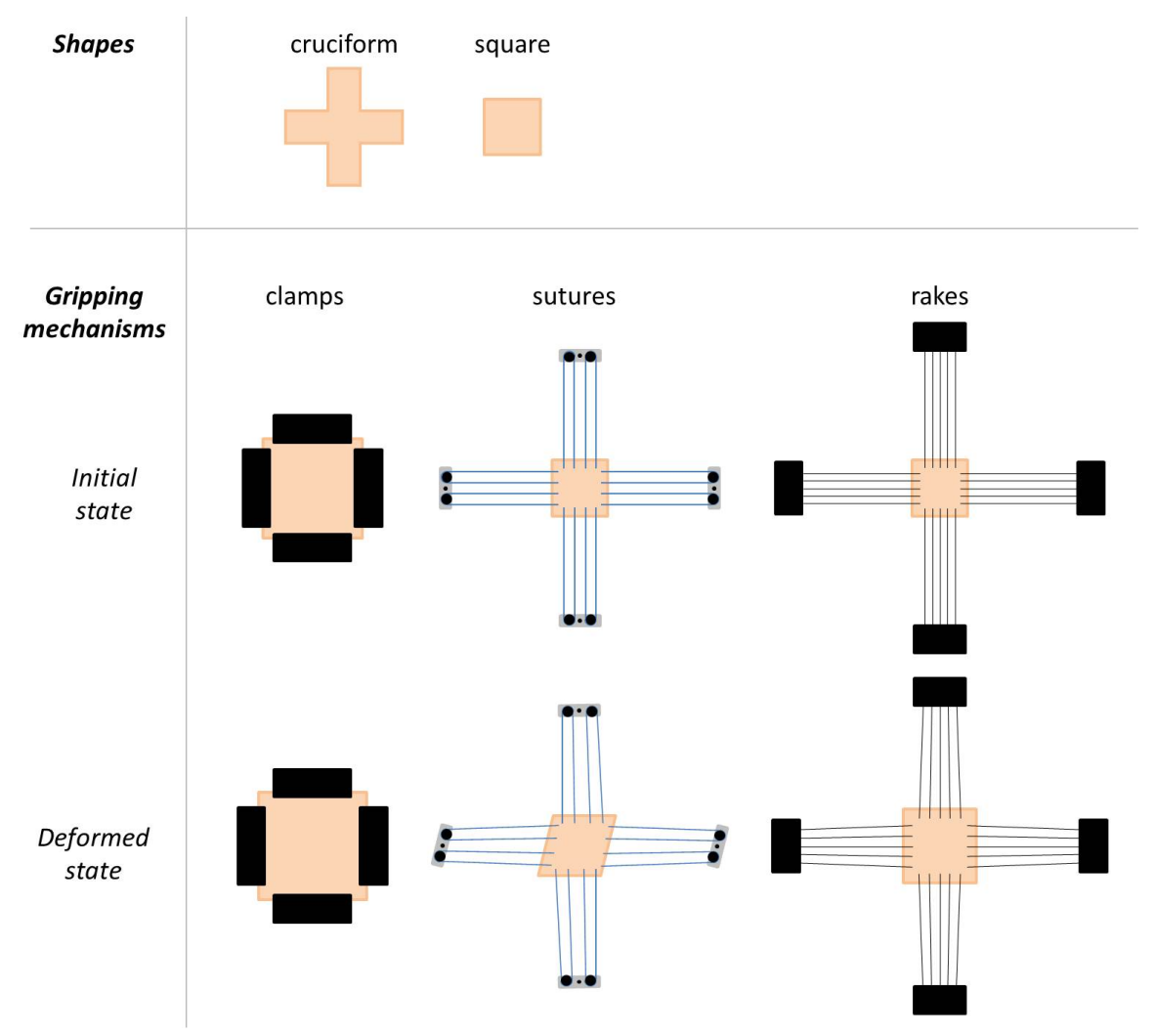

Figure 1: Different specimen shapes: cruciform and square; and different gripping mechanisms: clamps, sutures and rakes for planar biaxial experiments in initial and deformed state. 


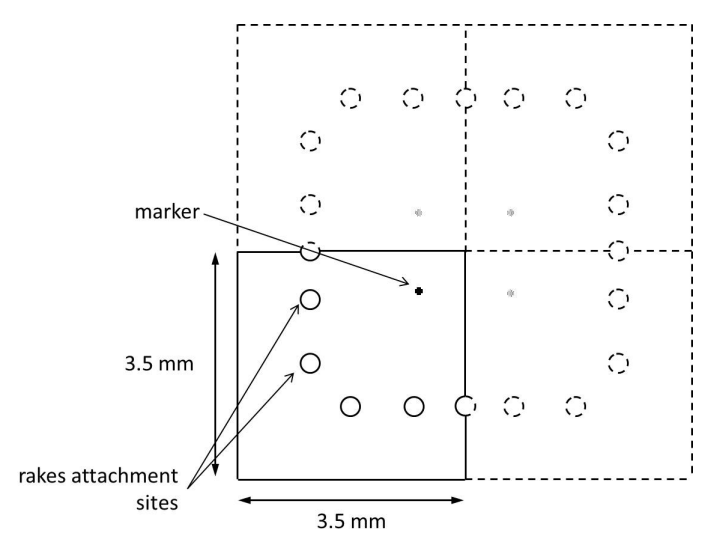

Figure 2: Due to symmetry, only a quarter of the testing specimen needs to be simulated. 


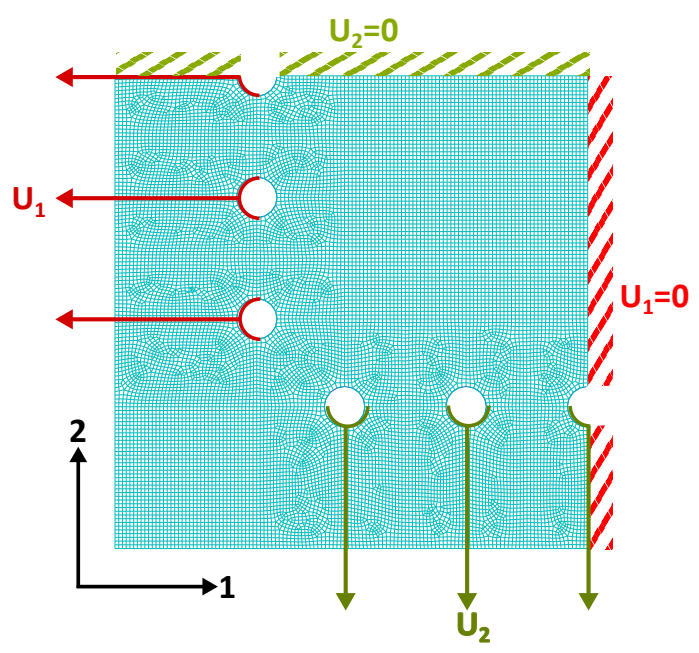

Figure 3: The FE mesh with the boundary and loading conditions. 


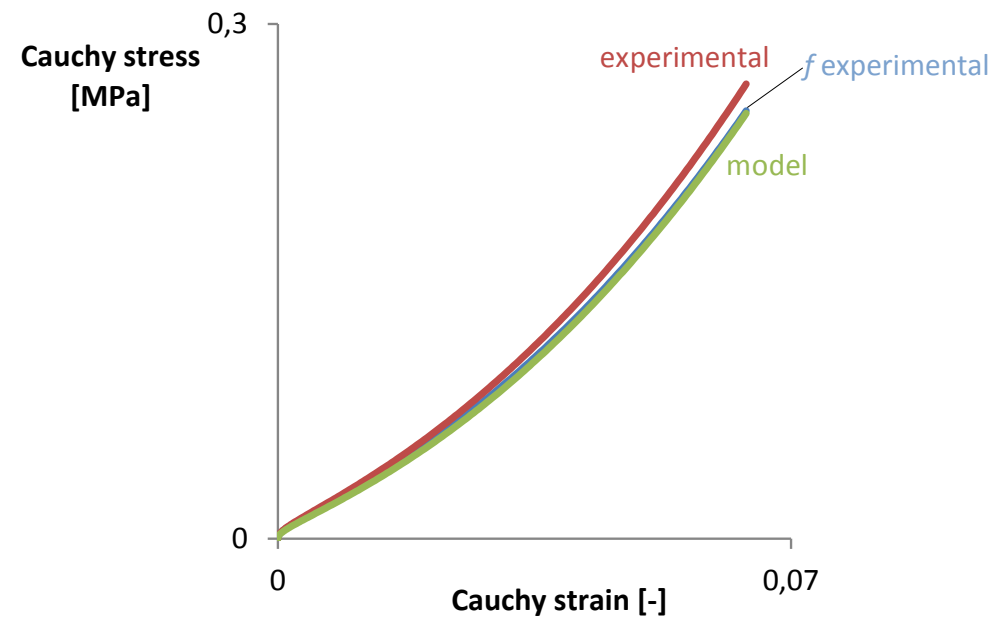

Figure 4: A representative figure in which the experimental stress is fitted on the model stress by means of a correction factor $f$. 


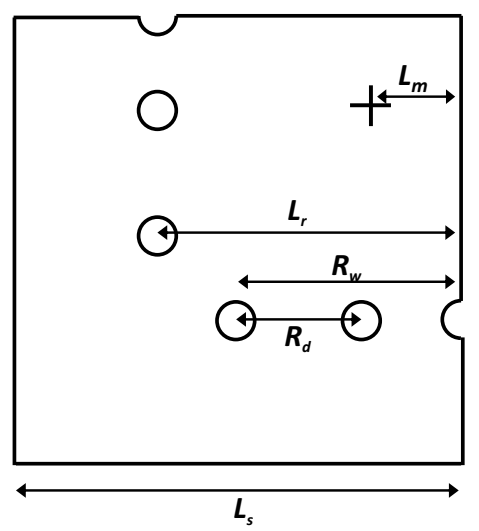

Figure 5: Definition of the geometrical parameters in a square quarter of a specimen. $L_{m}$ is the marker position, $L_{s}$ is the sample length, $L_{r}$ is the length covered by the rakes, $R_{w}$ is the rakes' width and $R_{d}$ is the inter-rake distance. Note that the specimen is symmetric and hence the dimensions in $\mathrm{x}$ and $\mathrm{y}$ direction are identical. 


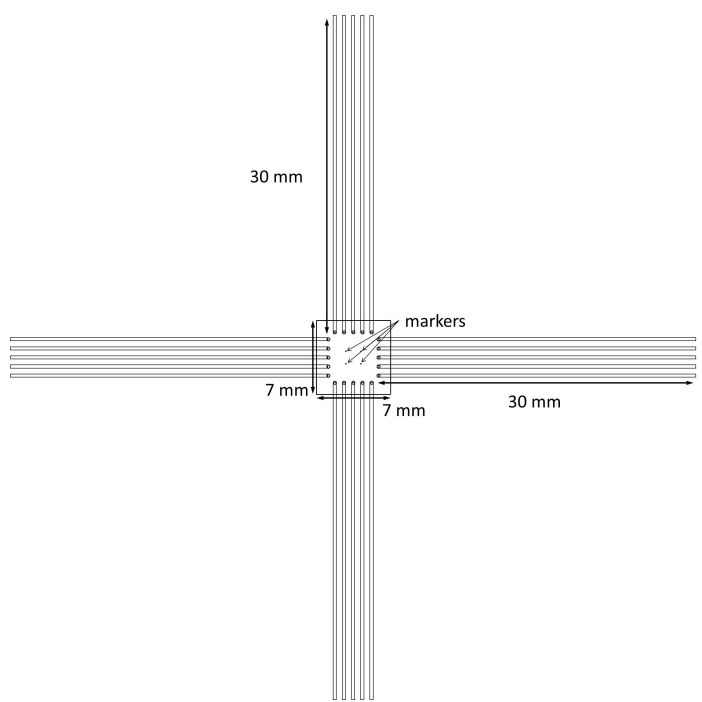

Figure 6: Dimensions of full sample and rakes and boundary conditions used in the FE model to verify the effect of shear. 


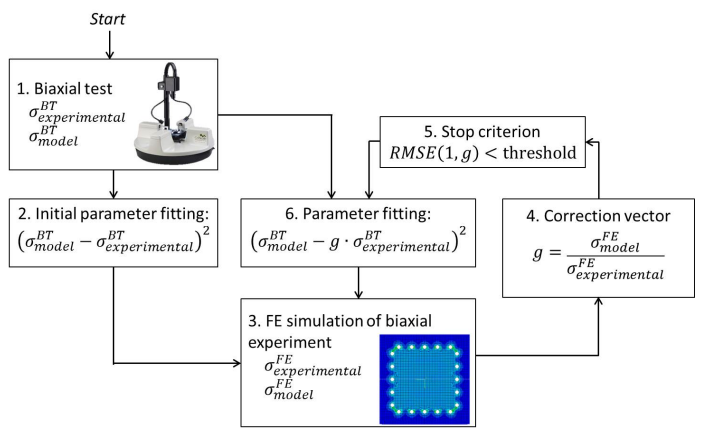

Figure 7: The IEC-parameter fitting procedure conducts a FE simulation with the material parameters from the classic procedure, determines a correction vector and subsequently performs a parameter fitting taking this correction vector into account. This process is repeated until a stop criterion is reached. 


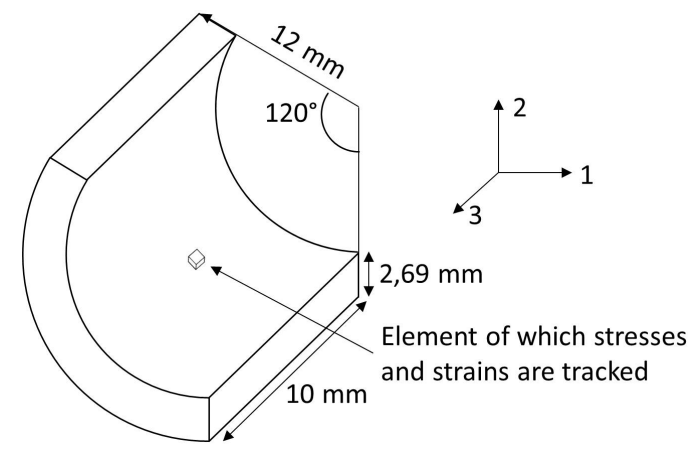

Figure 8: Initial geometry of a simplified human abdominal aorta. Due to symmetry only half of the cylindrical tube needs to be simulated. 

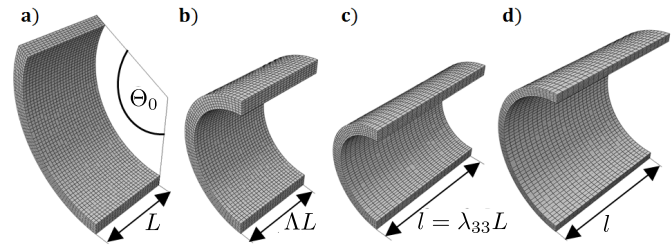

Figure 9: Loading of the simplified abdominal aorta is applied in consecutive steps: first, the segment is closed to form a cylinder (b), then an axial prestretch of $\lambda_{33}=1.08$ is applied (c) and finally the abdominal aorta is pressurized with a systolic pressure of $P_{\text {sys }}=126 \mathrm{mmHg}$ (d). 34 

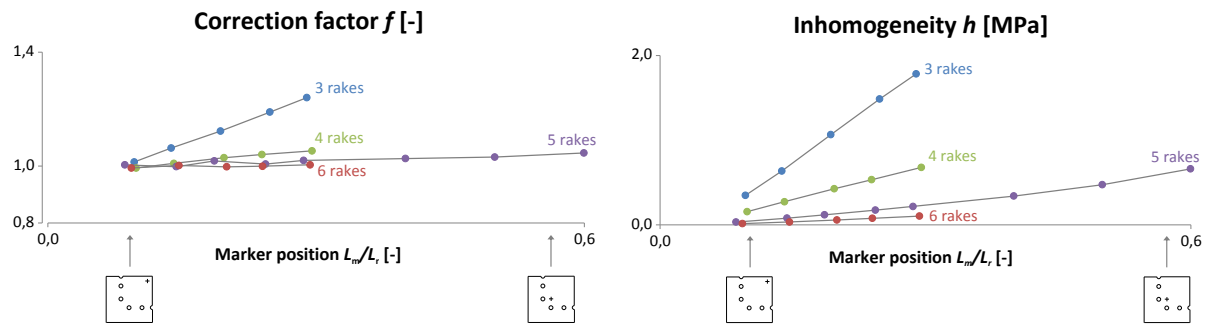

Figure 10: The correction factor $f$ and the inhomogeneity $h$ improve for an increasing number of rakes and a more central marker position $L_{m} / L_{r}$. The marker position $L_{m}$ is defined with respect to the length covered by the rakes $L_{r}$. Hence, 0 and 1 correspond to the marker at the specimen's center and at the rakes' attachment sites, respectively. 

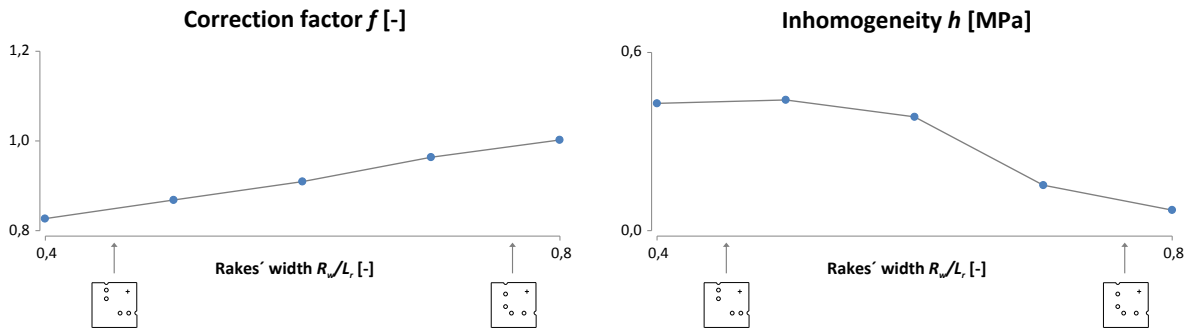

Figure 11: When loads are applied at a large section of the specimen edge, the correction factor $f$ improves and the homogeneity of the stress-strain field increases. The rakes' width $R_{w}$ is defined with respect to the length covered by the rakes $L_{r}$. 

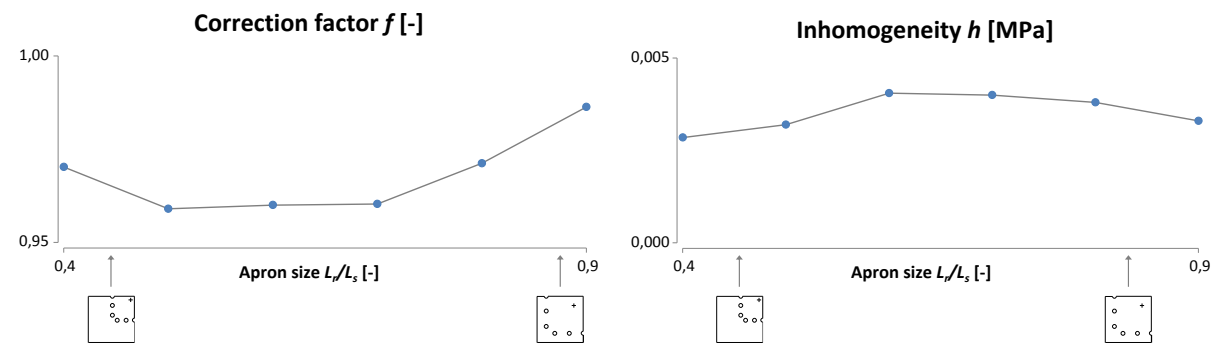

Figure 12: The correction factor $f$ and inhomogeneity $h$ are independent from the rake-tospecimen surface $L_{r} / L_{s}$ and thus from the size of the apron. 

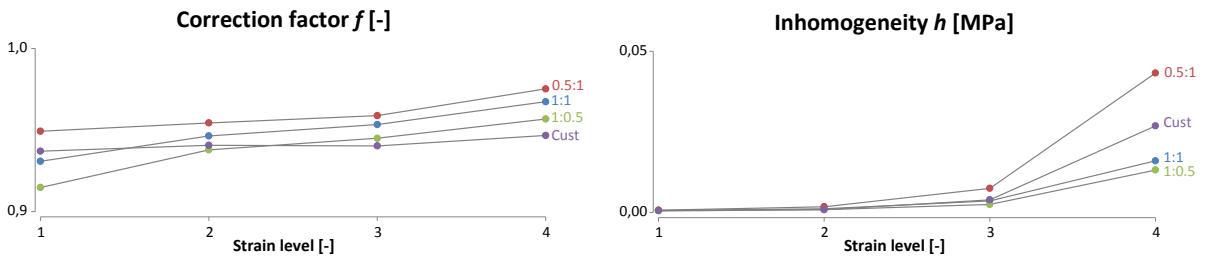

Figure 13: The correction factor $f$ and the inhomogeneity $h$ of the stress field both depend on the strain level and on the ratio. For this type of material and specimen orientation the $1: 0.5$ ratio provides the most homogeneous stress field, while the $0.5: 1$ ratio has $f$ closest to 1 . 

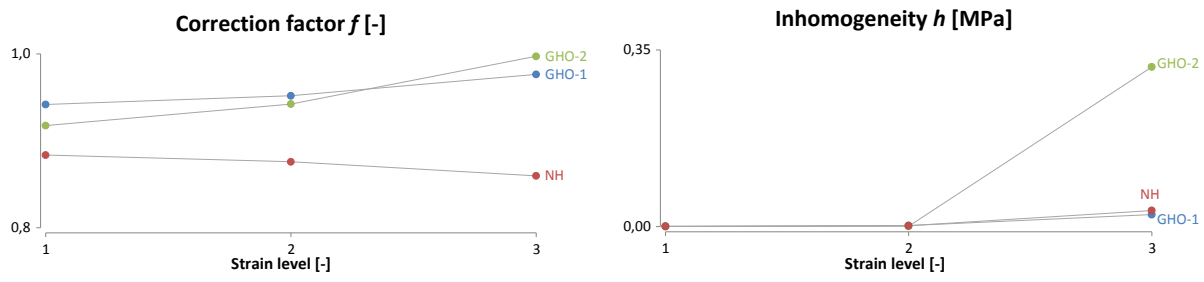

Figure 14: The correction factor $f$ and the inhomogeneity of the stress field $h$ depend on the material parameters and on the constitutive model. 

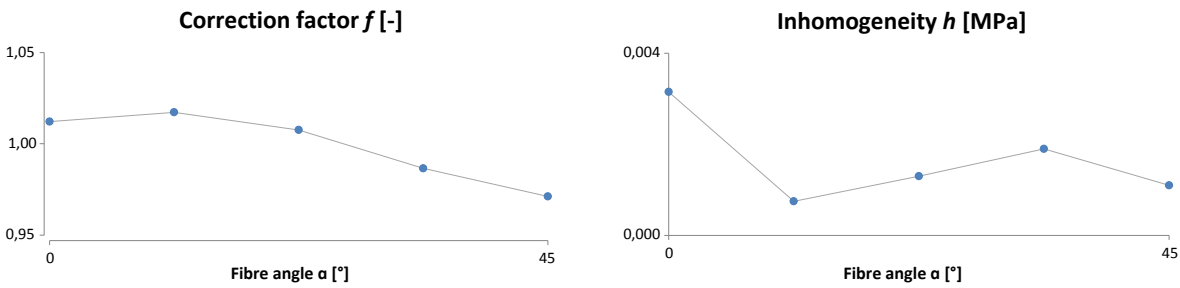

Figure 15: The fibre angle $\alpha$ has no significant effect on the correction factor $f$ and the inhomogeneity $h$ for this type of material. 


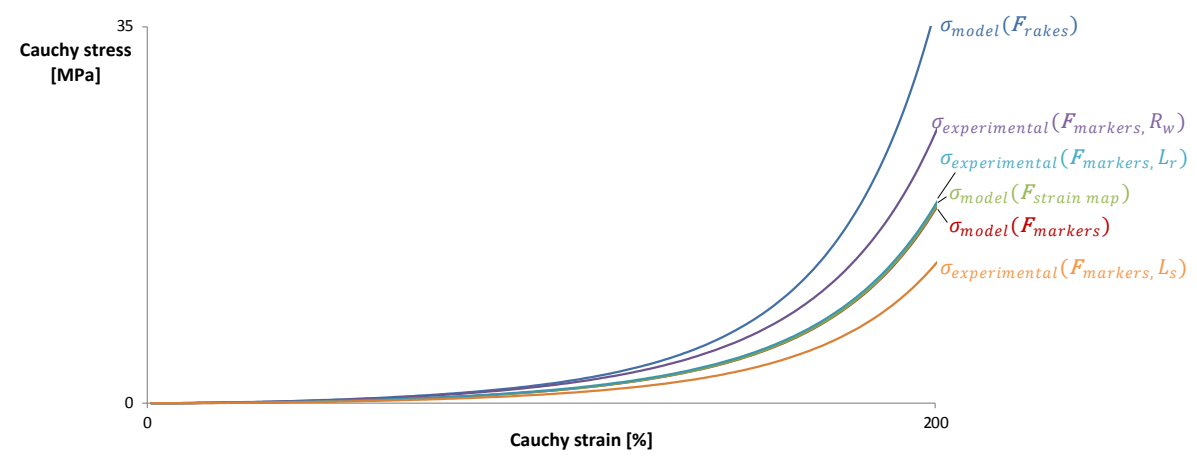

Figure 16: Different definitions of the deformation gradient tensor and section width result in different model and experimental stresses respectively. 


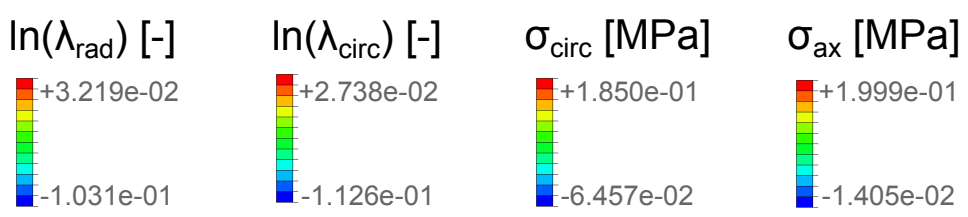

\section{Ground truth}

SL2

SL3

SL4

SW1

SW3

RM

SL2-IEC

SL3-IEC

\section{SL4-IEC}

Figure 17: Overview of the logarithmic strains $\left(\ln \left(\lambda_{\text {rad }}\right), \ln \left(\lambda_{\text {circ }}\right)\right)$ and Cauchy stresses $\left(\sigma_{\text {circ }}\right.$, $\left.\sigma_{a x}\right)$ for the different parameter sets as defined in Table 8 The SW1 and SW3 simulations show the largest deviations from the ground truth. 


\begin{tabular}{|c|c|c|c|c|c|}
\hline \multirow{2}{*}{\multicolumn{2}{|c|}{$\lambda_{11}: \lambda_{22}[\%]$}} & \multicolumn{4}{|c|}{ Strain levels } \\
\hline & & 1 & 2 & 3 & 4 \\
\hline \multirow{4}{*}{ 先 } & $1: 1$ & $6: 6$ & $12: 12$ & $24: 24$ & $36: 36$ \\
\hline & $0.5: 1$ & $3: 6$ & $6: 12$ & $12: 24$ & $18: 36$ \\
\hline & $1: 0.5$ & $6: 3$ & $12: 6$ & $24: 12$ & $36: 18$ \\
\hline & Custom & $6: 8$ & $12: 8$ & $24: 8$ & $36: 8$ \\
\hline
\end{tabular}

Table 1: The stretch values $\lambda_{11}$ and $\lambda_{22}$ [\%] for each ratio and every strain level. 


\begin{tabular}{l|ccccc} 
& $C_{10}[\mathrm{MPa}]$ & $k_{1}[\mathrm{MPa}]$ & $k_{2}[-]$ & $\alpha\left[{ }^{\circ}\right]$ & $\kappa[-]$ \\
\hline GOH-parameter set 1 & 0.019 & 5.15 & 8.64 & 38.8 & 0.24 \\
GOH-parameter set 2 & 0.017 & 0.56 & 16.21 & 51.0 & 0.18
\end{tabular}

Table 2: Different material parameter sets of the GOH-material model to introduce varying stiffness 


\begin{tabular}{l|c} 
& $C_{10}[\mathrm{MPa}]$ \\
\hline NH-parameter set 1 & 0.4 \\
NH-parameter set 2 & 1.0 \\
NH-parameter set 3 & 8.5
\end{tabular}

Table 3: Different material parameter sets of the NH-material model with the same linear stiffness as GOH-parameter set 1 for strain levels 1,2 and 3 . 


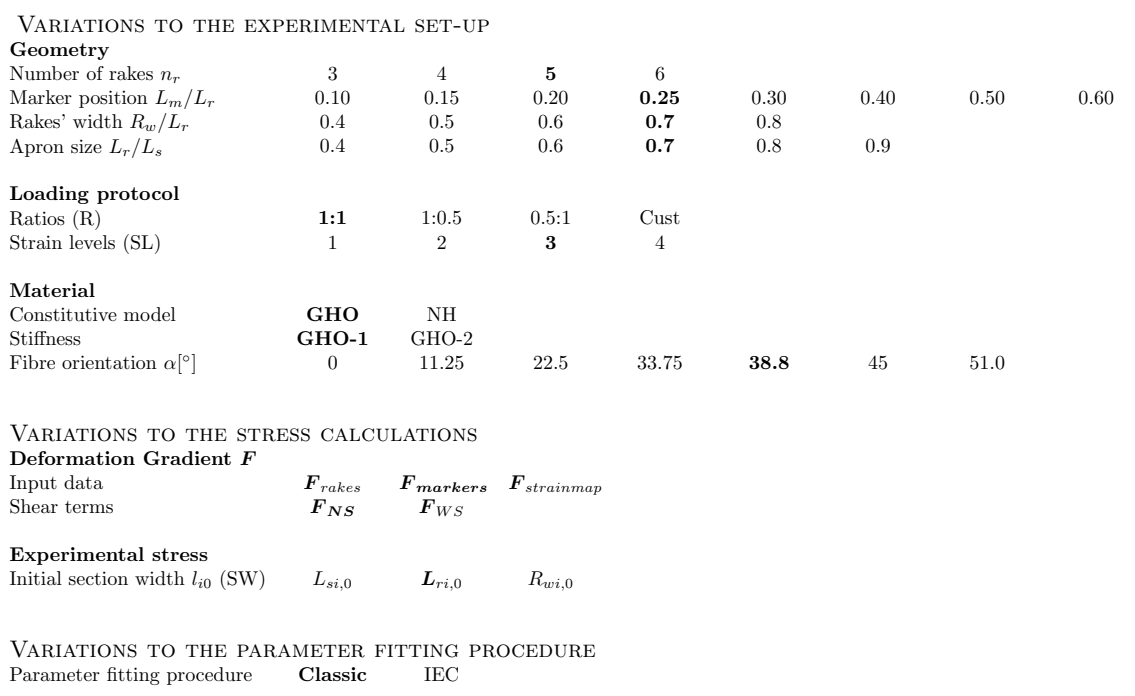

Table 4: An overview of all the parameters varied in the simulations and the data processing. The boldfaced values are the default ones. 


\begin{tabular}{l|cccc} 
Name & Ratio & Strain level & Initial section width $l_{i, 0}$ & Fitting procedure \\
\hline SL2 & $1: 1$ & 2 & $L_{r i, 0}$ & Classic \\
SL3 & $1: 1$ & 3 & $L_{r i, 0}$ & Classic \\
SL4 & $1: 1$ & 4 & $L_{r i, 0}$ & Classic \\
SW1 & $1: 1$ & 4 & $R_{w i, 0}$ & Classic \\
SW3 & $1: 1$ & 4 & $L_{s i, 0}$ & Classic \\
RM & $1: 1,0.5: 1,1: 0.5$ & 4 & $L_{r i, 0}$ & Classic \\
SL2-IEC & $1: 1$ & 2 & $L_{r i, 0}$ & IEC, 2nd iteration \\
SL3-IEC & $1: 1$ & 3 & $L_{r i, 0}$ & IEC, 2nd iteration \\
SL4-IEC & $1: 1$ & 4 & $L_{r i, 0}$ & IEC, 2nd iteration
\end{tabular}

Table 5: Overview of the different datasets, different definitions of initial section width $l_{i 0}$ and different fitting procedures used to obtain the different parameter sets. 


\begin{tabular}{l|ccccc|cc} 
NAME & $C_{10}[\mathrm{MPa}]$ & $k_{1}[\mathrm{MPa}]$ & $k_{2}[-]$ & $\alpha\left[^{\circ}\right]$ & $\kappa[-]$ & $\mathrm{RMSE}[\mathrm{MPa}]$ & $\mathrm{MPE}[\%]$ \\
\hline Ground truth & $\mathbf{0 . 0 1 9 0}$ & $\mathbf{5 . 1 5 0 0}$ & $\mathbf{8 . 6 4 0 0}$ & $\mathbf{3 8 . 8 0}$ & $\mathbf{0 . 2 4 0 0}$ & & \\
NS & 0.0727 & 6.7056 & 10.2000 & 37.57 & 0.2607 & 1.1468 & 71.21 \\
WS & 0.0729 & 6.7105 & 10.2042 & 37.56 & 0.2607 & 1.1468 & 71.41
\end{tabular}

Table 6: The parameters obtained through fitting taking shear into account (WS) or neglecting shear (NS), using the classic parameter fitting procedure. 


\begin{tabular}{c|c|ccccc|cc} 
& RATIO & $\boldsymbol{C}_{10}[\mathrm{MPa}]$ & $\boldsymbol{k}_{1}[\mathrm{MPa}]$ & $\boldsymbol{k}_{2}[-]$ & $\boldsymbol{\alpha}\left[^{\circ}\right]$ & $\boldsymbol{\kappa}[-]$ & $\mathrm{RMSE}[\mathrm{MPa}]$ & $\mathrm{MPE}[\%]$ \\
\hline Input & & $\mathbf{0 . 0 1 9 0}$ & $\mathbf{5 . 1 5 0 0}$ & $\mathbf{8 . 6 4 0 0}$ & $\mathbf{3 8 . 8 0}$ & $\mathbf{0 . 2 4 0 0}$ & & \\
Classic & $1: 1$ & 0.0651 & 6.1790 & 9.3304 & 37.59 & 0.2573 & 3.4115 & 56.19 \\
& $1: 1,0.5: 1,1: 0.5$ & 0.0687 & 6.0660 & 9.2624 & 37.75 & 0.2557 & 1.5208 & 59.16 \\
IEC-1 & $1: 1$ & 0.0123 & 5.1329 & 8.6350 & 38.87 & 0.2396 & 1.8018 & 7.20 \\
& $1: 1,0.5: 1,1: 0.5$ & 0.0128 & 5.1465 & 8.6452 & 38.85 & 0.2399 & 0.9088 & 6.59 \\
IEC-2 & $1: 1$ & 0.0200 & 5.1517 & 8.6407 & 38.79 & 0.2400 & 0.2286 & 1.07 \\
& $1: 1,0.5: 1,1: 0.5$ & 0.0201 & 5.1500 & 8.6397 & 38.80 & 0.2400 & 0.0291 & 1.16
\end{tabular}

Table 7: The new IEC-parameter fitting procedure shows a significant improvement in the result: the material parameters resemble the input parameters better than those obtained with the classic procedure. 


\begin{tabular}{l|ccccc|c} 
NAME & $C_{10}[\mathrm{MPa}]$ & $k_{1}[\mathrm{MPa}]$ & $k_{2}[-]$ & $\alpha\left[^{\circ}\right]$ & $\kappa[-]$ & $\mathrm{MPE}[\%]$ \\
\hline Ground truth & $\mathbf{0 . 0 1 9 0}$ & $\mathbf{5 . 1 5 0 0}$ & $\mathbf{8 . 6 4 0 0}$ & $\mathbf{3 8 . 8 0}$ & $\mathbf{0 . 2 4 0 0}$ & \\
SL2 & 0.0927 & 6.5932 & 10.5081 & 36.47 & 0.2682 & 91.06 \\
SL3 & 0.0389 & 5.7736 & 8.8697 & 38.30 & 0.2484 & 24.86 \\
SL4 & 0.0651 & 6.1790 & 9.3304 & 37.59 & 0.2573 & 56.19 \\
SW1 & 0.0889 & 8.4052 & 9.3281 & 37.60 & 0.2573 & 932.08 \\
SW3 & 0.0455 & 4.3253 & 9.3304 & 37.59 & 0.2573 & 34.76 \\
RM & 0.0687 & 6.0660 & 9.2624 & 37.75 & 0.2557 & 59.16 \\
SL2-IEC & 0.0248 & 5.2463 & 8.8348 & 38.62 & 0.2428 & 7.26 \\
SL3-IEC & 0.0201 & 5.1478 & 8.6376 & 38.81 & 0.2399 & 1.19 \\
SL4-IEC & 0.0200 & 5.1517 & 8.6407 & 38.79 & 0.2400 & 1.07
\end{tabular}

Table 8: The parameters obtained through fitting different datasets, using different definitions of initial section width $l_{i 0}$ and different fitting procedures (see Table 5) are shown. These parameter sets are used to investigate the consequences of suboptimal parameters. 


\begin{tabular}{l|rr|rr|rr|rr} 
& \multicolumn{2}{|c|}{$\boldsymbol{\sigma}_{\text {circ }}[\mathbf{k P a}]$} & \multicolumn{2}{c|}{$\boldsymbol{\sigma}_{a x}[\mathrm{kPa}]$} & \multicolumn{2}{|c}{$\boldsymbol{\lambda}_{\text {rad }}[\%]$} & \multicolumn{2}{c}{$\boldsymbol{\lambda}_{\text {circ }}[\%]$} \\
NAME & MAX & MIN & MAX & MIN & MAX & MIN & MAX & MIN \\
\hline Ground truth & $\mathbf{- 2 1 . 3}$ & $\mathbf{- 2 6 . 1}$ & $\mathbf{- 3 . 3}$ & $\mathbf{- 1 2 . 7}$ & $\mathbf{1 1 1 . 1 9}$ & $\mathbf{1 0 2 . 6 3}$ & $\mathbf{9 0 . 2 2}$ & $\mathbf{8 3 . 2 7}$ \\
SL2 & -53.6 & -66.9 & 8.4 & 7.3 & 105.73 & 101.38 & 91.32 & 87.57 \\
SL3 & -34.6 & -36.8 & -2.4 & -8.1 & 108.81 & 102.40 & 90.42 & 85.10 \\
SL4 & -45.3 & -53.2 & 1.3 & -0.8 & 107.03 & 101.93 & 90.84 & 86.51 \\
SW1 & -63.5 & -72.5 & 1.9 & 0.5 & 107.02 & 103.09 & 89.81 & 86.52 \\
SW3 & -31.1 & -37.2 & 0.9 & -2.5 & 107.03 & 100.30 & 92.32 & 86.50 \\
RM & -47.2 & -55.6 & 1.7 & -0.1 & 106.93 & 101.98 & 90.79 & 86.58 \\
SL2-IEC & -26.0 & -28.6 & -3.2 & -11.3 & 110.22 & 102.45 & 90.38 & 84.00 \\
SL3-IEC & -21.4 & -26.2 & -3.3 & -12.7 & 111.18 & 102.64 & 90.21 & 83.28 \\
SL4-IEC & -22.1 & -26.6 & -3.3 & -12.5 & 111.02 & 102.62 & 90.22 & 83.40
\end{tabular}

Table 9: The minimal and maximal Cauchy stresses $\left(\sigma_{c i r c} \& \sigma_{a x}\right)$ and stretch ratios $\left(\lambda_{\text {rad }}\right.$ $\left.\& \lambda_{\text {circ }}\right)$ for simulations with different material parameter sets. With respect to the ground truth, the largest deviations occur in SW1, SW3 and SL2. 